\title{
Conflitos Interparentais e Individuação em Jovens Adultos Portugueses: Papel dos Conflitos de Lealdade ${ }^{1}$
}

\author{
Catarina Pinheiro Mota ${ }^{2}$ \\ Universidade de Trás-os-Montes e Alto Douro \\ Paula Mena Matos \\ Faculdade de Psicologia e de Ciências da Educação da Universidade do Porto
}

\begin{abstract}
Resumo - O objetivo do presente estudo foi analisar em que medida os conflitos de lealdade e a triangulação a uma das figuras parentais medeiam a associação entre a percep̧̧ão de conflitos inter-parentais e o processo de individuação de jovens adultos. Adicionalmente, pretendeu-se testar se dimensões positivas da relação parental moderam a associação antes mencionada. Os participantes foram 538 jovens adultos portugueses entre os 18 e os 30 anos de idade, de ambos os gêneros. Para ambas as figuras parentais, a coligação (mas não a triangulação) medeia o efeito dos conflitos inter-parentais na individuação dos jovens, sendo que a percepção de cuidado recíproco e intimidade na relação parental moderam a relação entre conflitos inter-parentais e individuação dos jovens. Os resultados foram discutidos à luz do paradigma da vinculação e da formação da identidade.
\end{abstract}

Palavras-chave: conflito inter-parental, conflitos de lealdade, individuação, vinculação

\section{Interparental Conflicts and Individuation in Young Portuguese Adults: The Role of Loyalty Conflicts}

\begin{abstract}
The goal of the present study was to examine whether loyalty conflicts and pressure to side to one of the parents mediate the association between the perception of interparental conflicts and the individuation process in young adults. In addition, we wanted to test whether positive dimensions of the parental relationship moderate the above mentioned associations. Participants were 538 Portuguese man and women with ages between 18 and 30 years. The results indicate that coalition mediates the effect of interparental conflict on the individuation of the young adult, and that the perception of reciprocal care and intimacy in the parental relationship moderates the association between interparental conflicts and individuation. Results were discussed according to the attachment theory and the development of the identity of the young adult.
\end{abstract}

Key words: interparental conflict, loyalty conflicts, individuation, attachment

O processo de separação-individuação constitui um tema cuja abordagem tem sido cada vez mais enfatizada em torno do contexto físico e emocional que rodeia os jovens (Arnett, 2006). Desde a infância até à vida adulta, os jovens vão sendo progressivamente integrados num contexto exterior ao ambiente parental, criando oportunidades de experienciar e integrar novos desafios. Um passo crucial na transição da adolescência para a vida adulta representa a construção da identidade pessoal. De acordo com Erikson (1972), a constituição da identidade assume-se como uma tarefa desenvolvimental relevante que permite uma definição de quem é a pessoa, quais os valores e as direções que aspira seguir, criando um sentido de continuidade e de unicidade pessoal. Note-se que esse processo é em si pautado por uma necessidade de exploração do mundo emocional e cognitivo, em que os jovens reorganizam ideias, valores e sentimentos,

1 Apoio: Este trabalho é financiado por Fundos FEDER através do Programa Operacional Fatores de Competitividade - COMPETE e por Fundos Nacionais através da FCT - Fundação para a Ciência e a Tecnologia no âmbito do projeto FCOMP-01-0124-FEDER-022714

2 Endereço para correspondência: Universidade de Trás-os-Montes e Alto Douro. Departamento de Educação e Psicologia, Edifício do CIFOP, Rua Dr. Manuel Cardona, UTAD, Apartado 1013, 5001-558, Vila Real, Portugal.E-mail: catppmota@utad.pt seguindo-se um compromisso consigo mesmo, fruto de um investimento pessoal (Marcia, 1966).

Ao longo do processo de desenvolvimento, uma crescente separação é entendida à luz de um "afastamento" físico das figuras parentais que, posteriormente, se estende para a individuação e o desenvolvimento autônomo do self (Mattanah, Brand, \& Hancok, 2004). À luz da teoria da vinculação, um modelo do self como figura suscetível de ser amada associa-se positivamente a um modelo de figura de vinculação disponível e facilitadora de exploração (Ainsworth, 1991; Bowlby, 1988). Nessa medida, jovens que desenvolvem relações com os pais pautadas pela proximidade, responsividade e encorajamento à exploração face às suas necessidades desenvolvimentais, tendencialmente desenvolvem imagens mais positivas de si e dos demais. Uma importante fonte de crescimento pessoal decorre da diversidade de experiências de exploração proporcionadas ao longo do percurso de desenvolvimento, prevalecendo a garantia de retorno a uma base segura (Bowlby, 1988).

Assim, a qualidade dos laços estabelecidos entre os jovens e as figuras primárias de afeto deve ser entendida como um seguimento complementar ao processo de exploração. $\mathrm{Na}$ continuidade da revisão dos modelos internos dinâmicos ao longo da vida verifica-se que esses são passíveis de se alterar de acordo com a natureza e a qualidade das experiências 
que preenchem o percurso de desenvolvimento dos jovens (Ainsworth, 1991; Bowlby, 1988; Marrone, 1998) e se reportam no processo de individuação subsequente. A individuação é um processo evolutivo que ocorre na díade relacional entre pais e filhos, esse processo começa na adolescência e estende-se até à idade adulta. Podem-se diferenciar duas dimensões nucleares ao processo, nomeadamente a ligação e a individualidade (Youniss \& Smollar, 1985). A ligação aos pais é ainda assumida como sendo estável ao longo dos anos da infância, adolescência e até ao início da vida adulta num nível alto. Por sua vez a individualidade é caracterizada como a independência do jovem à autoridade parental, marcada pela mudança de autoridade unilateral para uma autoridade de cooperação e de reciprocidade, com tendência à progressão durante a adolescência e a jovem adultez (Mota \& Rocha, 2012). Scabini e Manzi (2011) acrescentam que um contexto familiar favorável, com relacionamentos de qualidade, propicia um processo de individuação bem sucedido.

De acordo com as dinâmicas realizadas ao longo do ciclo vital, Arnett (2006) e Bynner (2005) destacam a pertinência de reconhecer e analisar diferenças no processo de separação e individuação dos jovens adultos, sublinhando relevantes divergências entre os jovens oriundos de distintos contextos sócio-econômicos. Segundo Bynner (2005), existem fatores moderadores que reportam essas diferenças, nomeadamente, a qualidade da relação com as figuras parentais, as escolhas de carreira, as qualificações académicas e os fatores de empregabilidade. De acordo com essa ideia, destacamos, no presente estudo, aspectos que se prendem com fatores de vulnerabilidade e risco inerentes aos conflitos inter-parentais.

Os conflitos inter-parentais assumem uma relevância significativa no que concerne à compreensão do processo de desenvolvimento das crianças, adolescentes e jovens adultos. A literatura aponta para a associação entre os conflitos inter-parentais (na dinâmica educacional dos filhos) e implicações negativas no exercício do papel parental (e.g., Cummings \& Davies, 2002; Ellis, \& Boyan, 2010; Nigg et al., 2009). Esta questão traduz-se particularmente na diminuição da disponibilidade psicológica e da qualidade da responsividade na interação com os filhos, repercutindo-se, assim, também na natureza da vivência emocional dos jovens no seio da família (Pedro, Ribeiro, \& Shelton, 2012). Questionamos, no entanto, em que medida os conflitos inter-parentais, em si, afetam o bem-estar dos jovens adultos. A existência de conflitos inter-parentais moderados torna-se, em alguns casos, não só inevitável como uma oportunidade de desenvolvimento, no sentido de potenciar mudanças adaptativas no sistema familiar (Schnarch, 2000). Falamos de uma abordagem distinta dos conflitos inter-parentais, mais focalizada nas propriedades que o caracterizam, e que não consideram apenas a intensidade e a frequência com que ocorrem, mas também os modos como são abordados, expressos e, sobretudo, resolvidos. A percepção de que os pais não apenas apresentam discordâncias, mas também as discutem e as resolvem entre si, parece fazer uma importante diferença quando analisamos as implicações emocionais e o desenvolvimento do processo de adaptação psicossocial dos jovens (Mota \& Matos, 2009a). Numa perspetiva de resiliência, a percepção por parte dos jovens de resolução adaptativa dos conflitos interparentais mediante, entre outros aspectos, a regulação emocional adequada das divergências e do recurso a estratégias construtivas de resolução de problemas, pode ser um elemento fundamental. Esta questão coloca-se na medida em que facilita a organização das estruturas internas e a construção do conceito de si e dos outros, bem como a representação das relações entre os indivíduos. De acordo com Bretherton (2000), os jovens criam uma imagem de si como sendo figuras amadas que se prende com os modelos parentais de figuras de vinculação sensíveis, disponíveis e responsivas. Deste modo, ainda que os pais tenham divergências, o facto de as resolverem de forma adaptativa colocando e discutindo os diferentes pontos de vista com uma expressão emocional adequada, procurando a sua resolução torna-se um factor decisivo. A observação dos filhos desse processo parece facilitar, por um lado, uma maior estabilidade na dinâmica emocional da família, e por outro lado, uma aprendizagem acerca de uma forma adaptativa de resolver conflitos relacionais (Lindsey, Colwell, Frabutt, \& MacKinnon-Lewis, 2006; Siegel, 2010).

Por conseguinte, o desenvolvimento emocional dos jovens atravessa desafios permanentes, no qual se incluem os conflitos inter-parentais, todavia um vínculo seguro parece representar um elemento protetor que pode facilitar a adaptação (e.g., Claussen \& Crittenden, 2000). Ao invés, quando os conflitos inter-parentais não resolvidos continuam na sua frequência e intensidade poderão afetar a relação de vinculação com os filhos. Um aumento de interações negativas e a indisponibilidade psicológica dos pais poderá levar a que as necessidades emocionais dos jovens não sejam percebidas e respondidas (e.g., Cox, Paley \& Hater, 2001; Harold, et al. 2013; Pedro, Ribeiro, \& Shelton, 2012).

Alguns estudos sugerem que um estado emocional negativo derivado da exposição ao conflito inter-parental afeta o sentido de segurança emocional interna, diminuindo a autoestima e potenciando sentimentos de culpa e ameaça nos jovens (e.g., Buehler \& Welsh, 2009; Ellis, \& Boyan, 2010; Grych, Raynor, \& Fosco, 2004; Mota \& Matos, 2009b; Nigg et al., 2009). Nesta dinâmica, os jovens procuram acionar mecanismos no sentido de restabelecer a segurança emocional, pelo que o sentimento de pertença se torna fundamental. A necessidade de se manterem próximos das figuras de vinculação primárias poderá conduzir a processos de coligação e triangulação, sendo que a tomada de posição por uma das figuras parentais poderá acarretar conflitos de lealdade dificilmente geridos pelos jovens. Importa, pois, perceber em que medida os conflitos de lealdade podem interferir com o bem-estar dos jovens e, mais concretamente, com os processos de individuação que marcam o desenvolvimento emocional bem conseguido.

Minuchin (1974) perspetiva os conflitos de lealdade como processos em que os jovens são levados a escolher, devido a demandas, entre uma das figuras parentais, que os colocam inadvertidamente como que triangulados ou, por outras palavras "apanhados no meio" (caught in the middle). De acordo com o autor, a escolha de uma das figuras sugere estar contra a outra, pelo que os jovens que vivenciam esta estrutura disfuncional parecem sentir-se constrangidos. A triangulação constitui um processo em que a criança ou jovem se encontra envolvido na interação dos conflitos 
interparentais, sendo conduzidos a tomar partido, muitas vezes transportando mensagens entre os pais no sentido de minimizar ou evitar os conflitos (Minuchin, 1974). A coligação surge muitas vezes associada à triangulação, sendo mais frequente quanto mais jovem são os filhos; neste processo os jovens assumem uma aliança com uma das partes contra a outra, o que se traduz no desenvolvimento por parte dos jovens de uma ansiedade significativa (Buchanan \& Heiges, 2001). Neste sentido, alguns autores (e.g., Buchanan \& Heiges, 2001; Buchanan, Maccoby, \& Dornbusch, 1991; Lopez, 1991) sublinham que a triangulação e a coligação expressam um efeito negativo no bem-estar dos filhos, nomeadamente baixa autoestima, problemas comportamentais, depressão e ansiedade (Buchanan, Maccoby, \& Dornbusch, 1996; Ellis \& Boyan, 2010; Franck \& Buehler, 2007; Gerard, Buehler, Franck, \& Anderson, 2005; Nigg et al., 2009).

Mais recentemente, Swanson (2005) aponta no seu estudo realizado com 231 jovens universitários, que os conflitos de lealdade, nomeadamente a triangulação, a coligação e a percepção de envolvimento nos conflitos inter-parentais assumem um papel mediador entre a associação dos conflitos inter-parentais e o processo adaptativo dos jovens adultos, transversalmente à configuração familiar (intacta ou divorciada). Grych et al. (2004) ressaltam esta idéia, na medida em que adolescentes em famílias intactas e adolescentes em famílias divorciadas apresentaram-se similarmente afetados pela triangulação aquando da existência de conflitos inter-parentais.

A investigação sugere, ainda, que o controlo psicológico (associação que tem vindo a ser encontrada nas práticas parentais, nomeadamente nos comportamentos de alienação parental), não só faz com que os jovens se sintam triangulados, mas também serve como mediador entre os conflitos inter-parentais e os comportamentos de internalização dos jovens, nomeadamente, depressão e ansiedade (Johnston, Walters, \& Olesen, 2005). A esse propósito, Swanson (2005) enfatiza não só as consequências negativas que advêm da vivência de conflitos de lealdade, mas também que fatores podem potenciar os sentimentos dos jovens se sentirem divididos. A relação de coparentalidade parece fazer diferença nesse processo. Um dos maiores preditores dos conflitos de lealdade e particularmente de triangulação constitui a qualidade da relação de coparentalidade (Buchanan et al., 1991; Siegel, 2010). Entende-se por coparentalidade (coparenting, em inglês) a articulação entre os papéis parentais relativos ao cuidado geral dos filhos, incluindo valores, ideais e expectativas que são dirigidas aos mesmos, numa responsabilidade conjunta, entre pai e mãe, pelo seu bem-estar (Feinberg, 2002; Van Egeren \& Hawkins, 2004). De acordo com Buchanan et al. (1991), adolescentes menos susceptíveis de se sentirem triangulados na relação são aqueles cujas figuras parentais estão envolvidos numa relação coparental de suporte, cooperação e diálogo.

Por conseguinte, o presente estudo assenta no pressuposto de que a qualidade da ligação afetiva com as figuras parentais proporciona sentimentos de afeição e estabilidade na relação que conduzem ao processo de individuação (Buhl, 2008a). Todavia, alguns fatores parecem comprometer essa dinâmica, como são os processos inerentes aos conflitos inter-parentais, nomeadamente a forma como são percebidos pelos jovens em termos da sua resolução. Se os filhos se encontram no seio de disputas parentais poderão facilmente ser levados a condicionar a sua relação com uma das partes parentais, sendo conduzidos ao desenvolvimento de conflitos de lealdade (Siegel, 2010; Walper, Kruse, Noack, \& Schwarz, 2004). Nessa linha o presente estudo procura analisar em que medida os conflitos inter-parentais poderão exercer efeitos ao nível dos processos de individuação dos jovens, particularmente por via da experiência de conflitos de lealdade dos filhos aos pais, designadamente da experiência de coligação e de triangulação.

Embora a prática clínica assuma de forma clara uma compreensão desse processo, em geral e particularmente em Portugal são escassos ou inexistentes os estudos capazes de relacionar os conflitos interparentais e o desenvolvimento de conflitos de lealdade (nomeadamente coligação e triangulação), bem como o seu impacto no processo de individuação dos jovens em idade adulta. Nesta medida, atendendo a que os conflitos inter-parentais são passíveis de desempenhar um fator de risco para a dinâmica relacional entre pais e filhos (o que surge largamente descrito na literatura), o presente estudo procura introduzir a noção de que conflitos interparentais resolvidos poderão diminuir a coexistência de conflitos de lealdade na relação com os filhos (triangulação e coligação), diminuindo a interferência no processo de individuação dos jovens adultos.

Por outra parte, a literatura tem vindo a apoiar de forma consistente o papel protetor da qualidade da vinculação aos pais face a fatores de risco, especialmente na infância e adolescência. No presente estudo abordamos a relevância dessa temática nos jovens adultos analisando, à luz do modelo teórico da vinculação, a importância da continuidade das ligações afetivas na manutenção de uma base segura e desenvolvimento de uma individuação saudável.

Face aos objetivos propostos, espera-se que, independentemente da figura parental, exista um papel mediador da coligação e triangulação na associação entre o conflito inter-parental percebido e o processo de individuação dos jovens adultos. Ao mesmo tempo, espera-se que para cada uma das figuras parentais, as características positivas da relação parental (intimidade, satisfação e cuidado) moderem a associação entre o conflito inter-parental e o processo de individuação. Prevê-se que as variáveis positivas da relação parental enquanto fonte de segurança emocional aumentem a individuação bem-sucedida e a procura de manutenção do laço, e diminuem a negação das necessidades de vinculação, independentemente da existência de conflitos inter-parentais.

\section{Método}

\section{Participantes}

No estudo participaram 538 jovens adultos portugueses, $392(72,9 \%)$ a frequentar o ensino universitário, com idades compreendidas entre os 18 e os 30 anos $(\mathrm{M}=21,10$; DP = 2,93), 335 do género feminino $(62,3 \%)$ e $203(37,7 \%)$ do género masculino. Do total dos participantes, 515 (95,7\%) são solteiros e $318(71,4 \%)$ vivem com ambas as figuras parentais. 


\section{Instrumentos}

No âmbito do projeto internacional (YAGISSP - Young Adults in Germany, Italy, Sweden, Spain and Portugal) onde se insere o presente estudo foram usados os instrumentos que se seguem. Em alguns casos os instrumentos não foram usados na íntegra, apresentando-se compostos pelas dimensões mais significativas que vão ao encontro dos objetivos de estudo e que serão descritas seguidamente. Foi garantida a adequação das propriedades psicométricas de todos os instrumentos, por via da análise da consistência interna e da realização de análises fatoriais confirmatórias (com recurso ao programa EQS, versão 6.1).

\section{Conflito parental}

Os participantes relataram a sua percepção do conflito parental a partir do Childrens' Perception of Interparental Conflict Scale [Escala de Percepção de Conflitos Interparentais para Crianças] (CPIC; Grych, Seid, \& Fincham, 1992; Adaptação de Santos \& Matos, 2003). Embora o instrumento tenha originalmente 49 itens, com nove dimensões, no presente estudo foram respondidos apenas os itens referentes à frequência, que implica a frequência com que os conflitos se manifestam (três itens; "Os meus pais discutem") e resolução, que implica o grau de resolução adaptativa que os pais desenvolvem depois dos conflitos (um item "Após uma discussão, os meus pais voltam a fazer as pazes"), num total de quatro itens (sendo o item da resolução invertido por forma a avaliar a não resolução de conflitos inter-parentais). Cada item utiliza uma escala de tipo Likert, com cinco alternativas de resposta, que varia entre "Nunca e Sempre". A escolha dessas duas variáveis prendeu-se com a índole do estudo baseada na perceção de existência de conflitos e da forma como os pais reagem de forma mais ou menos adaptativa. As dimensões do instrumento utilizadas no presente estudo revelam consistência interna adequada com um alfa de Cronbach de 0,70. O instrumento tem sido usado em Portugal reportando resultados consistentes que nos garantem níveis adequados de validade e fiabilidade (Moura, Santos, Rocha, \& Matos, 2010), pelo que a dimensão conflitos interparentais surge agrupado apenas numa variável latente.

\section{Processo de Individuação}

A observação da individuação foi realizada mediante o Munich Individuation Test of Adolescence (MITA; Walper, Schwarz, \& Jurasic, 1996; Saraiva, Brandão \& Matos, 2010). $\mathrm{O}$ instrumento pretende avaliar aspetos normativos da individuação, abrangendo dimensões como a individuação bem sucedida, assim como problemas específicos inerentes ao processo desenvolvimental. É originalmente composto por 27 itens divididos em seis escalas, todavia no presente estudo foram apenas usadas três escalas: a individuação bem sucedida, que remete para um nível de individuação satisfatório do jovem para a idade esperada (quatro itens; "Os meus pais continuam a ser importantes para mim, apesar de eu seguir os meus próprios interesses"); a manutenção do laço, que se prende com a necessidade de manutenção saudável da proximidade com os pais (três itens; "Gostaria de viver coisas em conjunto com os meus pais"), e a negação das necessidades de vinculação, que representa um processo defensivo e de não aceitação da necessidade dos pais enquanto figuras significativas capazes de fornecer apoio (quatro itens; "É-me indiferente o que os meus pais pensam de mim"). Essas três dimensões compõem um fator de segunda ordem designado pelos autores de relacionalidade (relatedness). Cada item utiliza uma escala de tipo Likert com quatro alternativas de resposta, separadamente para pai e mãe, que varia entre "Nada de acordo" e "Totalmente de acordo". A consistência interna do instrumento foi medida através do alfa de Cronbach para a mãe e pai, respetivamente: individuação bem sucedida - $\alpha$ $=0,72 / 0,84$; manutenção do laço $-\alpha=0,59 / 0,69$; negação das necessidades de vinculação $-\alpha=0,65 / 0,70$. Foi realizada uma análise fatorial confirmatória de segunda ordem com a dimensão da relacionalidade (agrupando numa variável latente as três dimensões enquanto latentes e os respectivos itens enquanto variáveis observadas), sendo que os valores dos índices de ajustamento (MacCallum, Widaman, Preacher, \& Hong, 2001; Yuan, 2005) se encontram adequados, respetivamente para o pai e mãe $\left(\chi_{(8)}^{2}=197,94 ; p=0,001\right.$; $C F I=0,92 ;$ RMSEA $=0,09 ; \chi_{(5)}^{2}=167,55 ; p=0,001 ; C F I=$ 0,$93 ;$ RMSEA $=0,09$ ).

\section{Relação Parental}

A observação da qualidade da relação com as figuras parentais foi realizada mediante o Network Relationship Inventory (NRI, Fuhrman \& Buhrmester 1985). O inventário apresenta versões de avaliação para os pais, pares e par amoroso, tendo sido apenas utilizada a versão para pais. Originalmente é composto por 28 itens divididos em 8 dimensões, com uma escala de resposta de tipo Likert em cinco opções, que varia entre "Raramente ou Nunca e Quase sempre". Apenas foram utilizadas três dimensões do questionário, que avaliam características positivas da qualidade da relação com as figuras parentais: intimidade, que remete para a proximidade e temas partilhados pelos jovens com os pais (três itens; "Fala com os seus pais de coisas que não quer que os outros tenham conhecimento"); satisfação, que traduz a perceção de bem-estar na relação com os pais (dois itens; "Está satisfeito(a) na relação que tem com os seus pais?"); e cuidado, que atribui significado ao cuidado dado e percebido na relação com os pais (seis itens; "Sente amor e afeto pelos seus pais"). A consistência interna do instrumento foi medida através do alfa de Cronbach apresentando valores adequados para a mãe e pai, respetivamente: intimidade - $\alpha=0,89 / 0,86$; satisfação $-\alpha=0,76 / 0,79$; cuidado $-\alpha=0,94 / 0,96$. Foi realizada a análise fatorial confirmatória de segunda ordem com todas as dimensões do instrumento obtendo-se valores dos índices principais de ajustamento adequados (MacCallum et al., 2001; Yuan, 2005), respetivamente para o pai e mãe $\left(\chi_{(25)}^{2}=1010,5 ; p=0,001 ; C F I=0,94 ; R M S E A=0,06\right.$; $\left.\chi_{(27)}^{2}=1373 ; p=0,001 ; C F I=0,91 ; R M S E A=0,07\right)$. As três dimensões foram agrupadas no modelo de equações numa única variável latente. 


\section{Conflitos de Lealdade}

A observação dos conflitos de lealdade foi realizada mediante o Loyalty Conflict Scale (Wendt, Kroll, Beckh, Gerhard, \& Walper 2002). Trata-se de uma escala composta por 12 itens, divididos em duas dimensões: coligação, que remete para a maior proximidade e inclusivamente uma tomada de partido que os jovens são levados a desenvolver na relação com uma das figuras parentais (seis itens; "A minha mãe gostaria que eu gostasse mais dela do que do meu pai"); e a triangulação, que implica uma ambivalência dos jovens na relação com os pais conduzindo a incerteza e divisão acerca do estabelecimento de proximidade com os mesmos - (seis itens; "Sinto-me dividido entre os meus pais"), com uma escala de resposta de tipo Likert em cinco opções, que varia entre "Nunca e Sempre". O instrumento foi usado na íntegra revelando uma consistência interna adequada, medida através do alfa de Cronbach: coligação- $\alpha=0,80$; triangulação - $\alpha=0,85$. Foi realizada uma análise fatorial confirmatória de segunda ordem com as duas dimensões do instrumento obtendo-se valores adequados dos índices principais de ajustamento (MacCallum et al., 2001; Yuan, 2005), a saber, $\chi_{(50)}^{2}=222,4 ; p=0,001 ; C F I=0,95 ; R M S E A=0,08$.

\section{Procedimento e estratégia de análise de dados}

Trata-se de um estudo transversal sob o ponto de vista do seguimento temporal (também conhecido como seccional ou de prevalência), fazendo o levantamento simultâneo da exposição e do efeito, considerando-se por isso um estudo observacional (Olsen \& George, 2004). Nesta medida, a recolha dos dados foi realizada num só momento em universidades e escolas profissionais da área metropolitana da cidade do Porto. A aplicação teve lugar em tempo letivo, sendo apoiada pela equipa de investigação. Aquando da administração, foram apresentados os objetivos gerais do estudo, pelo que, tratando-se de uma administração coletiva, foram dadas instruções estandardizadas de esclarecimento no que diz respeito ao preenchimento dos questionários de auto-relato, realçando em todo momento o carácter sigiloso e confidencial da informação inerente aos questionários, assim como a índole voluntária da participação no estudo. $\mathrm{O}$ mesmo procedimento foi utilizado para a recolha dos dados inerentes aos jovens não universitários destacando-se a colheita de cariz individual e aleatória.

Os dados foram trabalhados procedendo-se a análises correlacionais e diferenciais, univariadas e multivariadas. Não se verificaram diferenças significativas entre jovens universitários e não universitários em todas as variáveis em estudo, pelo que a amostra foi usada como um único grupo. Foram levadas a cabo análises de acordo com modelos de equações estruturais (SEM; programa EQS 6.1.). Esta análise combina em si os construtos das análises fatoriais, análises de caminhos (path analyses) e regressões, compondo relações de causalidade e interdependência entre as variáveis, decompondo os efeitos totais em efeitos diretos e indiretos e testando os índices de ajustamento no modelo global (Sipley, 1999). Foram testados os efeitos indiretos/mediadores dos conflitos de lealdade analisando

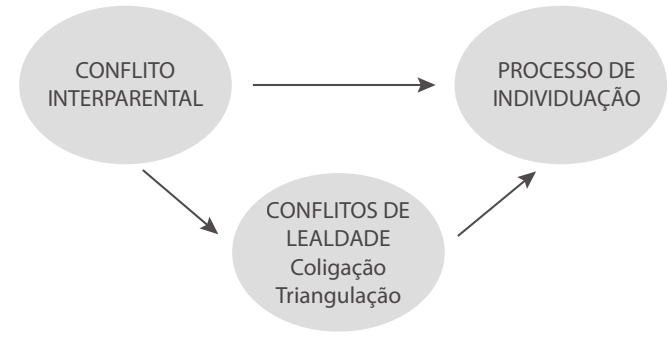

Figura 1. Modelo conceptual teórico representativo do efeito preditor dos conflitos interparentais e conflitos de lealdade no processo de individuação

a decomposição dos efeitos do modelo através dos valores $z$ para as variáveis relevantes com uma probabilidade acoplada de $95 \%$. Em análises adicionais, utilizou-se o teste de Wald à posteriori, para verificar o prejuízo da retirada de parâmetros não significativos para o valor do Qui-quadrado. Assim, no modelo final, apenas são considerados os parâmetros resultantes desta operação. Tratando-se de uma técnica confirmatória, mais do que exploratória, pretendeu-se determinar a validade do modelo conceptual construído neste estudo. O modelo testa em que medida a variável do conflito inter-parental prediz o processo de individuação dos jovens adultos. O modelo testa, ainda, o papel mediador dos conflitos de lealdade entre pais e filhos (coligação e triangulação) na associação anterior. Foi usado o método de máxima verosimilhança, tendo sido previamente confirmada a normalidade da amostra em estudo. O modelo hipotetizado para explicar a influência significativa da variável de conflitos inter-parentais na predição dos processos de individuação em jovens adultos encontra-se representado na Figura1.

\section{Resultados}

Papel mediador da coligação e triangulação na associação entre o conflito inter-parental e o processo de individuação

A análise do papel mediador da coligação e triangulação às figuras parentais foi realizada separadamente para pai $\mathrm{e}$ mãe como mostram as Figuras 2 e 3 . O modelo inicial teve em conta todas as relações possíveis entre as variáveis, isto é, as variáveis exógenas que definem os conflitos inter-parentais (nas suas dimensões de frequência e resolução) como preditoras quer dos conflitos de lealdade (coligação e triangulação), quer das três dimensões do processo de individuação (individuação bem sucedida, manutenção do laço e negação do desejo de vinculação). Do mesmo modo, os conflitos de lealdade seriam também preditores, em concomitância com a equação anterior, das dimensões da individuação.

Nesta medida, para a mãe o modelo de equações estruturais mostrou como os conflitos inter-parentais afetam de forma consistente e positiva o conflito de lealdade com os filhos, nomeadamente a coligação à mãe $(\beta=0,57)$ e a triangulação $(\beta=0,40)$. Para além disso, também observamos uma influência positiva da coligação à mãe na dimensão de triangulação $(\beta=0,25)$. O passo final permitiu-nos perceber como as variáveis se relacionam entre si, sendo interessante observar uma influência negativa da coligação à mãe no pro- 

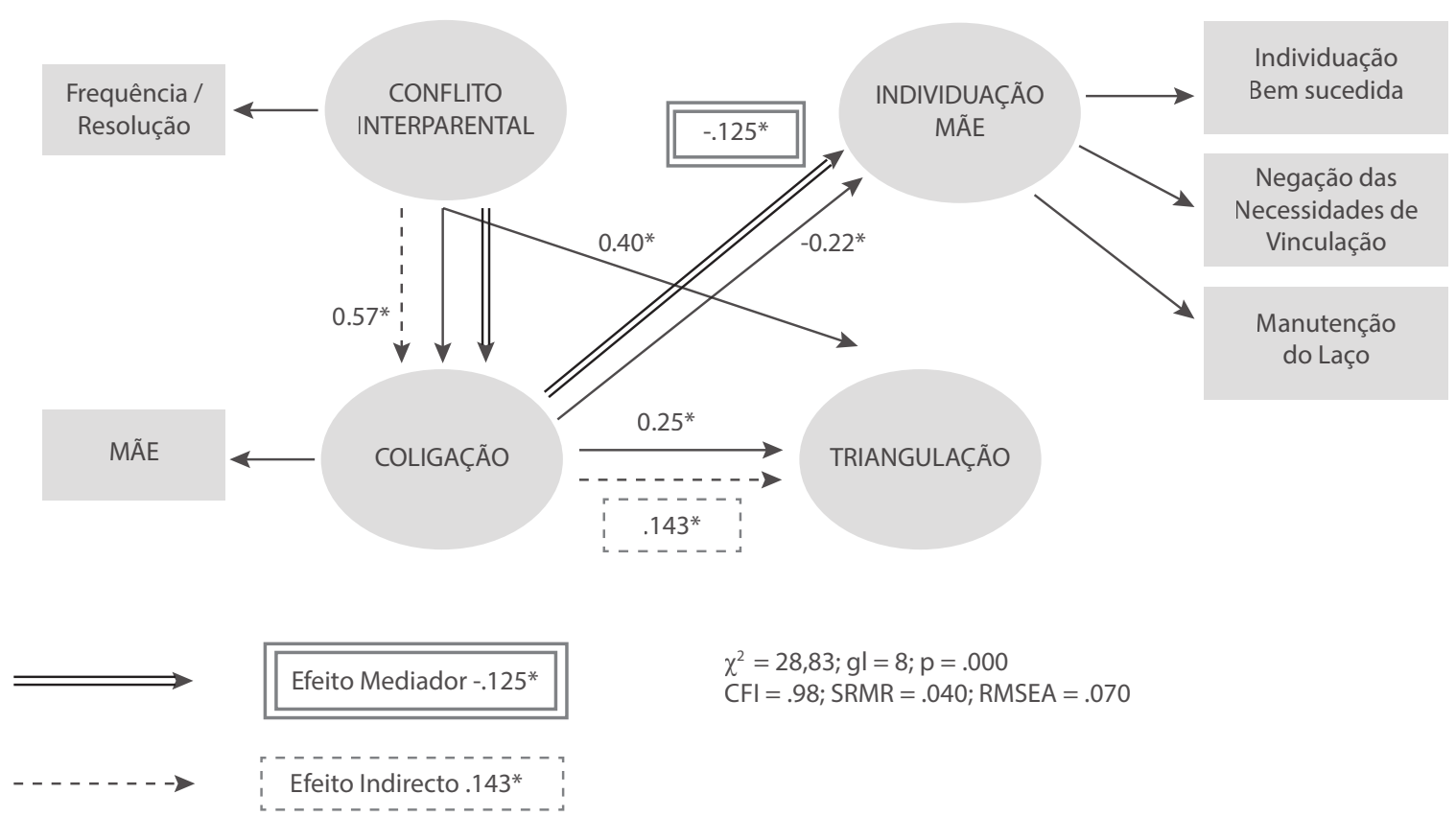

Figura 2. Modelo representativo do efeito mediador da coligação à mãe na associação entre os conflitos inter-parentais e o processo de individuação à mãe

cesso de individuação (composto pela individuação bem sucedida, a manutenção do laço e a negação das necessidades de vinculação) $(\beta=-0,22)$. Note-se que, tal como apresenta a Figura 2, observamos um papel mediador da coligação à mãe, pelo que os conflitos de lealdade, nomeadamente, a coligação mediou a influência dos conflitos inter-parentais no processo de individuação à mãe $(z=-4,777, E P=$ $0,026, p<0,05, \beta=-0,125)$. Ressaltamos, ainda, que os conflitos inter-parentais mostraram um efeito direto $(\beta=$ $.40)$ e indireto $(\mathrm{z}=5,080, E P=0,030, p<0,05, \beta=0,143)$ sobre a triangulação. Ao contrário do que seria esperado, a triangulação não exerceu um efeito mediador na associação entre o conflito parental e o processo de individuação à mãe. Os índices de ajustamento encontram-se adequados aos dados recolhidos $\left(\chi_{(8)}^{2}=28,83, p=0,001 ; C F I=0,98\right.$; $S R M R=0,040 ; R M S E A=0,070)$, viabilizando o modelo que está representado na Figura 2.

No que concerne à análise realizada com o pai, os resultados apresentam-se similares aos da mãe. Nesta medida observou-se igualmente que os conflitos inter-parentais afetam os conflitos de lealdade, com um efeito positivo na coligação ao pai $(\beta=0,61)$ e na triangulação $(\beta=0,38)$. Tal como acontece com a mãe, podemos observar um efeito mediador da coligação ao pai. Desta forma a coligação ao pai medeia de forma negativa a influência dos conflitos inter-parentais no processo de individuação ao pai $(z=-4,915$, $E P=0,030, p<0,05, \beta=-0,150)$. Paralelamente observamos que o conflito inter-parental influencia também a triangulação através da coligação ao pai, constatando-se um efeito indireto $(z=5,649, E P=0,023, p<0,05, \beta=0,161)$. Como se verificou com a mãe, no caso do pai, também a triangulação não desempenhou um papel mediador entre os conflitos inter-parentais e a individuação ao pai. Os índices de ajustamento encontram-se adequados ao que seria esperado $\left(\chi_{(8)}^{2}=36,11\right.$, $p=0,001 ; C F I=0,97 ; S R M R=0,060 ; R M S E A=0,082)$, tal como aponta o modelo da Figura 3.
Os resultados confirmam de forma parcial a primeira hipótese do estudo pelo que existe um papel mediador negativo da coligação na associação entre o conflito inter-parental percebido e o processo de individuação dos jovens adultos.

\section{Efeito moderador da relação parental na associação entre os conflitos inter-parentais e o processo de individuação}

No que concerne ao segundo objetivo do estudo, foram igualmente realizadas análises distintas para cada uma das figuras parentais. Os resultados foram desenvolvidos através de análises de variância univariada (ANOVA). Nesta medida, pretendeu-se analisar se, mesmo na presença de conflitos inter-parentais, haveria variáveis protetoras capazes de facilitar o processo de individuação. As análises foram desenvolvidas de acordo com a interação entre os conflitos inter-parentais (níveis altos e baixos) e as variáveis de individuação. Foram analisadas diferenças aquando da introdução das variáveis positivas da relação parental [(Conflitos inter-parentais X Relação parental) X Processo de Individuação], incluindo três variáveis para a relação parental (intimidade, satisfação e cuidado) e ainda três variáveis para o processo de individuação parental (individuação bem sucedida, manutenção do laço e negação das necessidades de vinculação).

Os resultados das análises de variância univariada, para a mãe, apresentaram diferenças significativas apenas em duas das interações, pelo que a dimensão de cuidado percebido na relação com a mãe parece afetar positivamente a individuação bem sucedida face à mãe $[F(1,529)=3,877 ; p=0,049]$. Nesta medida, constatou-se que, quando existe elevado conflito inter-parental, uma perceção de elevado cuidado materno se associa a níveis superiores de individuação. Ainda para a mãe, constatou-se uma diferença significativa face ao cuidado materno na dimensão da negação das necessidades 


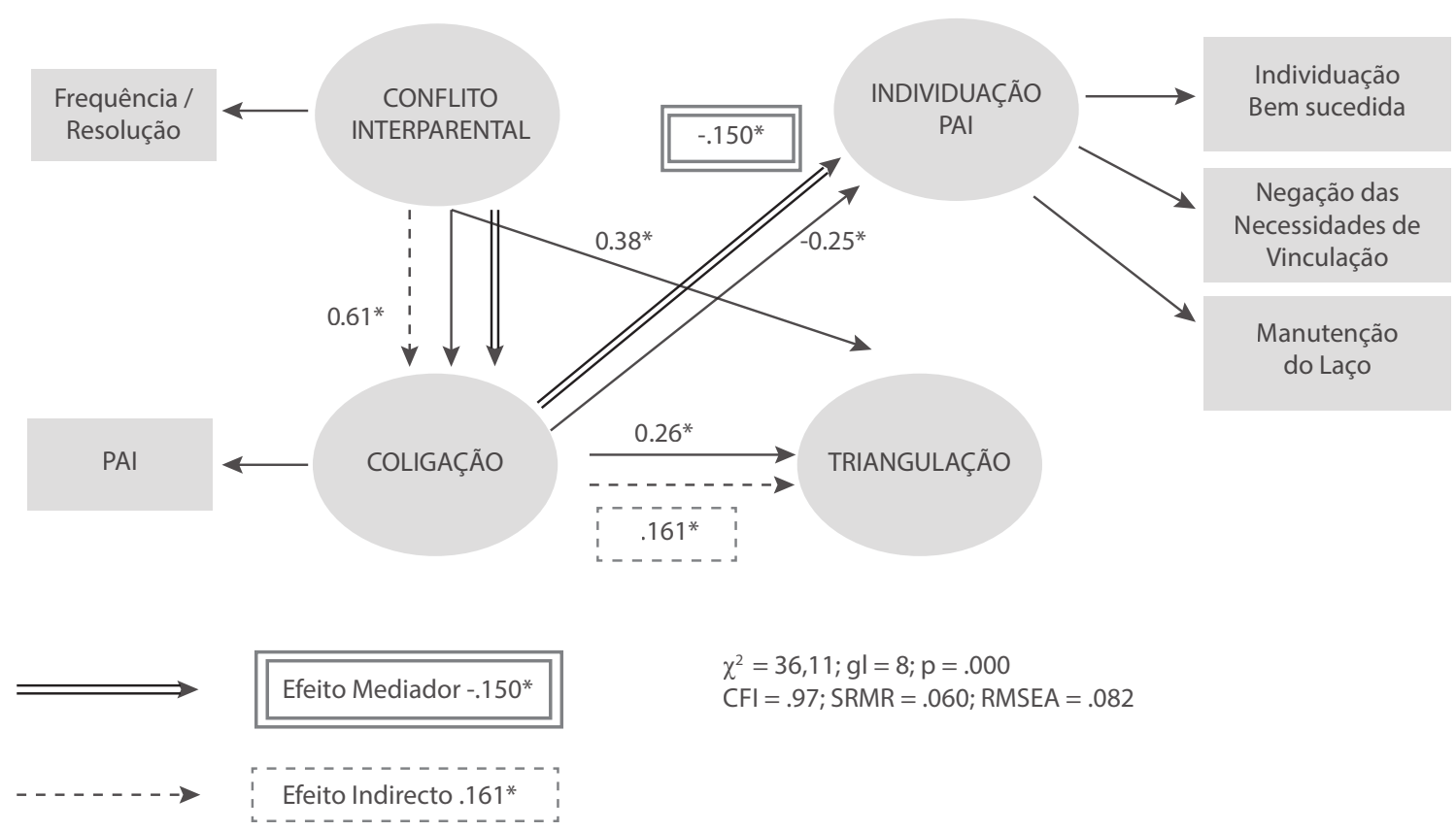

Figura 3. Modelo representativo do efeito mediador da coligação ao pai na associação entre os conflitos inter-parentais e o processo de individuação ao pai

de vinculação $[F(1,529)=5,235 ; p=0,023]$. Desta feita, quando existe um elevado conflito inter-parental, a percepção de elevado cuidado materno associa-se a uma menor negação das necessidades de vinculação materna quando comparado com níveis baixos de cuidado materno. Face às análises de variância univariada realizadas com o pai observaram-se diferenças significativas para duas interações. Numa primeira análise verificou-se que na presença de conflitos inter-parentais, a percepção de níveis elevados de intimidade e de cuidado na relação com o pai se associam a uma menor negação do desejo de vinculação ao pai, quando comparados com níveis inferiores de intimidade e níveis inferiores de cuidado, respectivamente $[F(1,529)=5,526 ; p=0,019] \mathrm{e}$ $[F(1,529)=5,714 ; p=0,017]$. Constatamos nesta medida que os resultados apoiam a segunda hipótese, na medida em que existe um papel moderador da relação parental, ainda que apenas a partir das dimensões de cuidado e intimidade em ambas as figuras parentais.

\section{Discussão}

O modelo teórico que serve de base ao presente estudo traduz a ideia de que os conflitos inter-parentais exercem um efeito significativo no desenvolvimento dos jovens. Os resultados obtidos relativamente ao primeiro objectivo corroboram parcialmente a hipótese inicialmente colocada indo ao encontro da literatura mais recente que assume uma significativa associação entre os conflitos inter-parentais e os processos de individuação (e.g., Buhl, 2008a, 2008b; Pedro, Ribeiro, \& Shelton, 2012; Walper \& Schwarz, 2001). Desta feita, constatamos que os conflitos inter-parentais poderão interferir com o processo de individuação bem sucedida no que respeita a cada uma das figuras parentais. As hipóteses do presente estudo previam uma associação positiva entre os conflitos inter-parentais e os conflitos de lealdade. Tal como apontam os resultados, para ambas as figuras parentais, esta hipótese é confirmada, observando-se uma associação positiva entre os conflitos inter-parentais e os conflitos de lealdade, nomeadamente a triangulação e a coligação. Atendendo a que os conflitos inter-parentais podem condicionar o processo de individuação dos jovens, testou-se o papel mediador dos conflitos de lealdade nesta associação. Os nossos resultados corroboram parcialmente esta hipótese, na medida em que apenas a dimensão da coligação exerceu um papel mediador, aumentando o poder de explicação entre os conflitos inter-parentais e a individuação dos jovens a ambas as figuras parentais. No âmbito de problemas de ajustamento, e designadamente de comportamentos de internalização e externalização, Fosco e Grych (2008) salientaram os efeitos adversos dos conflitos inter-parentais. Os autores apontaram como mediador desta associação o desenvolvimento de conflitos de lealdade entre os jovens e os pais, mais precisamente processos de triangulação que, juntamente com perturbações emocionais e baixa autoestima, conduziriam a um menor ajustamento. Franck e Buehler (2007) também sublinharam esta perspetiva na medida em que as hostilidades inter-parentais assumiram um efeito significativo no desenvolvimento de conflitos de lealdade exercendo um papel mediador negativo na predição do ajustamento dos jovens. Buehler e Welsh (2009) observaram que a triangulação se encontrava associada à internalização de problemas nos jovens (como ansiedade e depressão), variável que se revelou direta e significativamente associada aos conflitos inter-parentais e indisponibilidade parental.

Note-se que a coligação e a triangulação, embora conceitos próximos, parecem assumir significados distintos para os jovens, tal como reportam nos resultados do presente estudo, uma vez que os conflitos inter-parentais assumem um efeito indireto, através da coligação, na triangulação. Por conseguinte, os conflitos inter-parentais conduzem a uma coligação e triangulação dos jovens, todavia a coligação (ou a lealdade a 
uma das figuras parentais em detrimento da outra) parece ser um fio condutor para que os jovens se "sintam apanhados no meio" (feeling caught in the middle), ou o que se designa por triangulação (e.g. Buchanan \& Waizenhofer, 2001; Walper \& Schwarz, 2001).

Ressaltamos todavia que os conflitos inter-parentais, por si só, não implicam necessariamente uma interferência na relação entre os pais e os jovens adultos. Os conflitos (no limiar de um respeito mútuo) podem inclusive ser importantes no processo de crescimento pessoal dos jovens, no sentido em que são percebidas formas de lidar com a diferença e meios de resolução construtiva através de argumentos de negociação (Grych \& Fincham, 1990; Krishnakumar \& Buehler, 2000, Mota \& Matos, 2011; Schnarch, 2000). Esta relação não sugere uma associação causal, todavia sabemos que as dificuldades na resolução de conflitos por parte das figuras parentais são passíveis de condicionar a necessidade dos jovens recriarem um sentido de pertença e proteção que pode ser, paradoxalmente, encontrado na relação de coligação. Jovens que se sentem condicionados pelas escolhas dos pais e entrosados nos conflitos inter-parentais não resolvidos facilmente são conduzidos a tomar partido por uma das partes, o que traduz sentimentos de angústia e culpa inerentes à triangulação (Amato \& Afifi, 2006).

Nesta sequência, a segunda parte dos resultados aponta justamente para a importância da qualidade da relação parental na individuação dos jovens adultos. Retomando o modelo concetual do presente estudo, partiu-se da hipótese de que a qualidade relacional com as figuras parentais poderia exercer um papel moderador na associação entre os conflitos inter-parentais e o processo de individuação. A forma como os jovens percebem este ambiente relacional é fundamental para a elaboração dos seus esquemas emocionais, traduzindo um forte impacto na formação das ligações de vinculação futuras (Miljkovitch, 2004). Assim, corroborando esta hipótese, constatou-se que para ambas as figuras parentais, uma percepção de elevado cuidado parental mesmo na presença de um elevado conflito, modera os efeitos negativos no processo de individuação, nomeadamente prevenindo ou evitando sentimentos de rejeição e retração que poderiam revelar-se através da negação das necessidades de vinculação. Esta última dimensão pode ser entendida à luz de uma forma defensiva de lidar com o sofrimento emocional, numa expressão de autossuficiência que nem sempre corresponde às necessidades dos jovens adultos. A mesma situação foi observada no que concerne à intimidade com o pai. Deste modo, uma elevada intimidade na relação com o pai diminui o efeito negativo dos conflitos inter-parentais no processo de individuação, nomeadamente no evitamento das necessidades de vinculação. De acordo com alguns estudos (e.g., Buhl, 2008a, 2008b), a intimidade está estreitamente relacionada com a qualidade da ligação às figuras parentais, variável que de acordo com os autores surge positivamente correlacionada com o processo de individuação.

Walper, Kruse, Noack e Schwarz (2004) salientam que os conflitos inter-parentais sugerem um menor suporte e sensibilidade materna, com uma predição negativa da qualidade da relação estabelecida com a figura materna e um maior sentimento de triangulação por parte dos jovens. Note-se que, tal como já havíamos comentado, os conflitos inter-parentais não são necessariamente considerados uma interferência no processo de individuação, sempre que não surjam coligações derivadas da forma como os pais lidam com os conflitos. Walper e Schwarz (2001) apontam que, quando sentem mais segurança na sua própria relação com os companheiros, os pais parecem sentir menor necessidade de alianças com os filhos, o que se traduz num efeito significativamente positivo no processo de individuação.

Como nota final, julgamos oportuno apontar algumas das limitações que pautaram o presente estudo. Desta feita, tratando-se de um estudo transversal sob o ponto de vista do seguimento temporal, a amostra foi recolhida apenas num momento comprometendo a discussão de efeitos causais nas relações. Por outro lado, a amostra foi recolhida maioritariamente nas universidades, e com jovens estudantes, em geral a viverem em famílias intactas, pelo que a amostra não resulta completamente aleatória. Do mesmo modo, a recolha de informação foi realizada através de instrumentos de auto-relato, por si susceptíveis a fatores de enviesamento da informação, pelo que seria de todo pertinente, em futuros estudos, realizar recolhas de outros informantes, nomeadamente a perspetiva dos pais. Tornar-se-ia ainda pertinente a testagem da dinâmica dos conflitos inter-parentais e o processo de individuação noutras configurações familiares (famílias divorciadas ou monoparentais). Seria interessante equacionar outras formas de análise das variáveis, como sendo a testagem do efeito do processo de individuação na qualidade da relação parental, assumindo assim um carácter bidirecional.

\section{Referências}

Ainsworth, M. D. S. (1991). Attachments and other affectional bonds across the life cycle. In C. M. Parkes, J. StevensonHinde, \& P. Marris (Eds.), Attachment across the life cycle (pp.33-51). New York, NY: Routledge.

Amato, P. R., \& Afifi, T. D. (2006). Feeling caught between parents: Adult children's relations with parents and subjective wellbeing. Journal of Marriage and Family, 68, 222-235.

Arnett, J. J. (2006). Emerging adulthood in Europe: A response to Bynner. Journal of Youth Studies, 9, 111-123.

Bowlby, J. (1988). A secure base: Parent-child attachment and healthy human development. London: Basic Books.

Buchanan, C. M., \& Waizenhofer, R. (2001). The impact of interparental conflict on adolescent children: Considerations of family systems and family structure. In A. Booth, A. C. Crouter, \& M. Clements (Eds.), Couples in conflict (pp. 149-160). Mahwah, NJ: Erlbaum.

Buchanan, C. M., \& Heiges, K. L. (2001). When conflict continues after the marriage ends: Effects of post-divorce conflict on children. In J. H. Grych, \& F. D. Fincham (Eds.), Interparental conflict and child development: Theory, research, and applications (pp. 337-362). Cambridge, United Kingdom: Cambridge University Press.

Buchanan, C. M., Maccoby, E. E., \& Dornbusch, S. M. (1991). Caught between parents: Adolescents' experience in divorced homes. Child Development, 62, 1008-1029.

Buchanan, C. M., Maccoby, E. E., \& Dornbusch, S. M. (1996). Adolescents after divorce. Cambridge, MA: Harvard University Press. 
Buehler, C., \& Welsh, D. P. (2009). A process model of adolescent's triangulation into parent's marital conflict: The role of emotional reactivity. Journal of Family Psychology, 23, 167-180.

Buhl, H. M. (2008a). Development of a model describing individuated adult child-parent relationships. International Journal of Behavioural Development, 32, 381-389.

Buhl, H. M. (2008b). Significance of individuation in adult childparent relationships. Journal of Family Issues, 29, 262-281.

Bynner, J. (2005). Rethinking the youth phase of the life-course: The case for emerging adulthood? Journal of Youth Studies, $8,367-384$.

Cox, M. J., Paley, B., \& Hater, C. (2001). Interparental conflict and parent - child relationship. In J. H. Grych \& F. D. Fincham (Eds.), Interparental conflict and child development: Theory, research and application (pp. 249-272). Cambridge: Cambridge University Press.

Cummings, E. M., \& Davies, P. (2002). Effects of marital conflict on children: Recent advances and emerging themes in process-oriented research. Journal of Child Psychology and Psychiatric, 43, 31-63.

Ellis, E. M., \& Boyan, S. (2010). Intervention strategies for parent coordinators in parental alienation cases. American Journal of Family Therapy, 38, 218-236.

Erikson, E. H. (1972). Identidade, juventude e crise. Rio de Janeiro, RJ: Zahar.

Feinberg, M. E. (2002). Coparenting and the transition to parenthood: A framework for prevention. Clinical Child and Family Psychology Review, 5, 173-195.

Fosco, G. M., \& Grych, J. H. (2008). Emotional, cognitive, and family systems mediators of children's adjustment to interparental conflict. Journal of Family Psychology, 22, 843-854.

Franck, K. L., \& Buehler, C. (2007). A family process model of marital hostility, parental depressive affect, and early adolescent problem behaviour: The roles of triangulation and parental warmth. Journal of Family Psychology, 21, 614-625.

Furman, W., \& Buhrmester, D. (1985). Children's perceptions of the personal relationships in their social networks. Developmental Psychology, 21, 1016-1024.

Gerard, J. M., Buehler, C., Franck, K., \& Anderson, O. (2005). In the eyes of the beholder: Cognitive appraisals as mediators of the association between interparental conflict and youth maladjustment. Journal of Family Psychology, 19, 376-384.

Geuzaine, C., Debry, M., \& Liesens, V. (2000). Separation from parents in late adolescence: The same for boys and girls? Journal of Youth and Adolescence, 29, 79-91.

Greene, A. L., \& Grimsley, M. D. (1990). Age and gender differences in adolescents' preferences for parental advice: Mum's the world. Journal of Adolescent Research, 5, 396-413.

Grych, J. H., \& Fincham, F. D. (1990). Marital conflict and children's adjustment: A cognitive-contextual framework. Psychological Bulletin, 108, 267-90.

Grych, J. H., Seid, M., \& Fincham, F. D. (1992). Assessing marital conflict from the child's perspective: The children's perception of interparental conflict scale. Child Development, 63, 558-572.

Grych, J. H., Raynor, S. R., \& Fosco, G. M. (2004). Family processes that shape the impact of interparental conflict on adolescents. Development and Psychopathology, 16, 649-665.
Harold, G., Leve, L., Elam, K., Thapar, A., Neiderhiser, J., Ntsuaki, M., ...\& Reiss, D. (2013). The nature of nurture: Disentangling passive genotype-environment correlation from family relationship influences on children's externalizing problems. Journal of Family Psychology, 27(1) 12-21.

Johnston, J. R., Walters, M. G., \& Olesen, N. W. (2005). Is it alienating parenting, role reversal or child abuse? A study of children's rejection of a parent in child custody disputes. Journal of Child Custody, 5,191-218.

Krishnakumar, A., \& Buehler, C. (2000). Interparental conflict and parenting behaviors: A meta-analytic review. Family Relations, 49, 25-44.

Levpuscek, M. P. (2006). Adolescent individuation in relation to parents and friends: Age and gender differences. European Journal of Developmental Psychology, 3, 238-264.

Lindsey, E. W., Colwell, M. J., Frabutt, J. M., \& MacKinnon-Lewis, C. (2006). Family conflict in divorced and non-divorced families: Potential consequences for boys' friendship status and friendship quality. Journal of Social and Personal Relationships, 23, 45-63.

Lopez, F. G. (1991). Patterns of family conflict and their relation to college student adjustment. Journal of Counseling \& Development, 69, 257-260.

MacCallum, R. C., Widaman, K. F., Preacher, K. J., \& Hong, S. (2001). Sample size in factor analyses: The role of model error. Multivariate Behavioral Research, 36, 611-637.

Marcia, J. E. (1966). Development and validation of ego-identity status. Journal of Personality and Social Psychology, 3, 551-558.

Marrone, M. (1998). Attachment and interaction. London: Jessica Kingsley Publishers.

Mattanah, J. F., Brand, B. L., \& Hancok, G. R. (2004). Parental attachment, separation-individuation, and college student adjustment: A structural equation analysis of meditational effects. Journal of Counseling Psychology, 51, 213-225.

Mayseless, O., \& Scharf, M. (2009). 'Too close for comfort: Inadequate boundaries with parents and individuation in late adolescent girls'. American Journal of Orthopsychiatry, 79, 191-202.

Miljkovitch, R. (2004). A vinculação ao nível das representações. In N. Guedeney, \& A. Guedeney (Eds.), Vinculação: Conceitos e aplicação, Lisboa: Climepsi Editores.

Minuchin, S. (1974). Families and family therapy. Cambridge, Massachusetts: Harvard University Press.

Mota, C. P., \& Matos, P. M. (2011). Adolescência e conflitos parentais: Uma perspetiva de resiliência. In P. M. Matos, C. Duarte, \& M. E. Costa (Eds.), Famílias: Questões de desenvolvimento e intervenção (pp.125-151). Porto: LivPsic.

Mota, C. P., \& Matos, P. M. (2009a). Clinical implications of interparental conflict: Intervention with adolescents from different family contexts. Comunicação apresentada no 40th Society for Psychotherapy Research (SPR) International Meeting. Santiago do Chile, Chile.

Mota, C. P., \& Matos, P.M. (2009b). Vinculação, conflito e autoestima em adolescentes de famílias intactas e divorciadas. Psicologia: Reflexão e Crítica, 22, 317-325.

Mota, C. P., \& Rocha, M. (2012). Adolescência e jovem adultícia: Crescimento pessoal, separação-individuação e o jogo das relações. Psicologia: Teoria e Pesquisa, 28, 357-366. 
Moura, O., Santos, R. A., Rocha, M., \& Matos, P. M. (2010). Portuguese version of the Children's Perception of Interparental Conflict Scale (CPIC): Factor structure and invariance across adolescents and emerging adults. International Journal of Testing, 10, 364-382.

Nigg, J. T., Nikolas, M., Miller, T. S., Alexander, B., Klump, K., \& Von Eye, A. (2009). Factor structure of children's perception of interparental conflict scale for studies of youths with externalizing behavior problems. American Psychological Association, 21), 450-456.

Noller, P., \& Callan, V. J. (1990). Adolescents' perceptions of the nature of their communication with parents. Journal of Youth and Adolescence, 19, 349-362.

Olsen, C. \& George, D. (2004). Cross-sectional study design and data analysis: The young epidemiology scholars program (YES). Princeton, NJ: The Robert Wood Johnson Foundation.

Pedro, M. F., Ribeiro, T., Shelton, K. H. (2012). Marital satisfaction and partners' parenting practices: The mediating role of coparenting behavior. Journal of Family Psychology, 26, 50922. doi: $10.1037 / \mathrm{a} 0029121$.

Santos, R. A., \& Matos, P. M. (2003). The children's perception of interparental conflict scale. (Adaptação do instrumento não publicado). Faculdade de Psicologia e de Ciências da Educação da Universidade do Porto.

Saraiva, L., Brandão, T., \& Matos, P. M. (2010). Factor structure of the Portuguese version of MITA. Comunicação apresentada no Paper presented at the $5^{\text {th }}$ Congress of the European Society on Family Relations. Milão, Itália. Retrieved from http://www. yagiss.de/en/downloads/ESFR_2010_Saraiva_Matos.pdf

Scabini, E. (2000). Parent-child relationships in Italian families: Connectedness and autonomy in the transition to adulthood. Psicologia: Teoria e Pesquisa, 16, 23-30.

Scabini, E., \& Manzi, C. (2011). Family processes and identity. In S. J. Schwartz, K. Luyckx, \& V. L. Vignoles (Eds.), Handbook of identity theory and research (Vol. 2, pp. 565-584). New York: Springer.

Schnarch, D. (2000). Desire problems: A systemic perspective. In S. Leiblum \& R. Rosen (Eds.), Principles and practices of sex therapy (pp. 101146). New York: Guilford Press.

Shipley, B. (1999). Testing causal explanations in organismal biology: causation, correlation and structural equation modelling. Oikos, 86, 374- 382.
Siegel, J. P. (2010). A good-enough therapy: An object relations approach. In A. S. Gurman (Ed.), Clinical casebook of couple therapy (pp. 134-152). New York, NY: Guilford Press.

Swanson, J. A. (2005). Covert processes: Loyalty conflicts, child involvement, and parental alienation as mediators of the link between interparental conflict and college student adjustment. (Dissertação de mestrado não publicada). Faculty of Miami University. Department of Psychology. Oxford, Ohio.

Van Egeren, L. A., \& Hawkins, D. P. (2004). Coming to terms with coparenting: Implications of definition and measurement. Journal of Adult Development, 11, 165-178.

Walper, S., \& Schwarz, B. (2001). Adolescent's individuation in East and West Germany: Effects of family structure, financial hardship, and family processes. American Behavioral Scientist, 44, 1937-1954.

Walper, S., Kruse, J., Noack, P., \& Schwarz, B. (2004). Parental separation and adolescents' felt insecurity with mothers: Effects of financial hardship, interparental conflict, and maternal parenting in East and West Germany. Marriage \& Family Review, 36, 115-145.

Walper, S., Schwarz, B., \& Jurasic, S. (1996). Entwicklung und Erprobung des Münchner Individuations tests. Berichte aus der Arbeitsgruppe "Familienentwicklung nach der Trennung" 8/1996. Ludwig-Maximilians- Universität Munchen.

Wendt, V., Kroll, S., Beckh, K., Gerhard, K., \& Walper, S. (2002). Dokumentation der Erhebugsinstrumente der 4. Hauptbefragung. Berichte aus der Arbeitsgruppe, "FamilienEntwicklung nach der Trennung" 42/2002. LudwigMaximilians- Universitat München und Friedrich-SchillerUniversität Jena.

Youniss, J., \& Smollar, J. (1985). Adolescent relations with mothers, fathers and friends. Chicago: University of Chicago Press.

Yuan, K. H. (2005). Fit indices versus test statistics. Multivariate Behavioral Research, 40, 115-148.

Recebido em 15.02.2012

Primeira decisão editorial em 26.04.2013

Versão final em 10.05.2013

Aceito em 13.05.2013 